East African Medical Journal Vol. 77 No. 3 March 2000

CRANIOVERTEBRAL JUNCTION ANOMALIES SEEN AT KENYATTA NATIONAL HOSPITAL, NAIROBI

N.J.M. Mwang'ombe, MMed, PhD, Lond. and G.K. Kirongo, MMed, Surg., Department of Surgery, Kenyatta National Hospital, College of Health Sciences, University

of Nairobi, P.O. Box 19076, Nairobi, Kenya

\title{
CRANIOVERTEBRAL JUNCTION ANOMALIES SEEN AT KENYATTA NATIONAL HOSPITAL, NAIROBI
}

\author{
N.J.M. MWANG'OMBE and G.K. KIRONGO
}

\begin{abstract}
Objective: To study the frequency, mode of presentation and outcome following surgery of anomalies of the craniovertebral junction at the Kenyatta National Hospital.

Design: A retrospective study

Setting: Kenyatta National Hospital, Nairobi.

Subjects: Thirty eight patients with craniovertebral junction anomaly who were treated at the Kenyatta National Hospital.

Results: Thirty eight patients were diagnosed to have craniovertebral junction anomaly at the Kenyatta National Hospital between January 1988 and December 1994. Thirty of those patients underwent surgery. The ages ranged between 10 years and 49 years with a peak in the third decade. The male to female ratio was $2: 1$. The common mode of presentation was weakness of the extremities $(90 \%)$ followed by sensory disturbances such as numbness of the extremities (30\%), headache (30\%) and neck pain $(20 \%)$. The common clinical findings were ataxia, muscle wasting, sensory loss and brisk deep tendon reflexes. Most of the patients had computerised tomography (CT) scanning (80\%) done. Other radiological investigations which were done were plain $x$-rays of the skull and cervical spine $(\mathbf{5 0} \%)$, myelographic studies $(36 \%)$ and tomographic studies $(\mathbf{1 2 \%})$. Forty percent of the patients improved following surgery while further neurological deterioration was prevented in $48 \%$. There was an immediate post-operative mortality of $8 \%$.

Conclusion: Craniovertebral junction anomaly is an important clinicopathological, condition common amongst certain ethnic groups found in high altitude areas of the Kenya. Surgery may cause clinical improvement in $40 \%$ of the patients, mainly those who have been symptomatic for less than three years.
\end{abstract}

\section{INTRODUCTION}

Craniovertebral junction is the region formed by the occipital bone surrounding the foramen magnum and the upper two cervical vertebra. Within this bony and ligamentous enclosure is the spinal cord, medulla oblongata and the inferior portion of the cerebellum(1). Skeletal anomalies that give rise to symptoms in this area are basilar impression, occipitalisation of the atlas, odontoid process abnormalities and atlanto-axial dislocation. Neuromeningeal anomalies in this region include ArnoldChiari malformation, syringomyelia and basal arachnoiditis. The clinical presentation may be in the form of spastic tetraparesis and paraesthesia due to high cervical cord compression, cerebellomedullary syndromes causing ataxia, nystagmus, dizziness, vertigo and lower cranial nerve palsies or a combination of both. The aims of surgical treatment are decompression of neural structures, fusion of unstable joints and re-opening of obstructed cerebrospinal fluid (CSF) pathways.

\section{MATERIALS AND METHODS}

Hospital records of all patients with craniovertebral junction anomalies who were treated at the Kenyatta National Hospital between January 1988 and December 1994 were reviewed. The data was analysed to obtain age and sex distribution, ethnic background, clinical presentation, investigations done and diagnosis, surgical treatment given and outcome following treatment.

\section{RESULTS}

Thirty eight patients with craniovertebral junction anomalies were seen at Kenyatta National Hospital between January 1988 and December 1994. Thirty of these patients underwent surgery. Males were more common than females by a ratio of 2:1. Most of the patients were between the age of twenty and forty years (Figure 1). Most of the patients were from the Kikuyu (68\%), Meru (12\%) and Kamba $(8 \%)$ tribes of Kenya which are found in the high altitude Central and Eastern Provinces of the country. No cases were reported from the Luo and Luhyia tribal groups.

Other factors apart from the ethnic background may also be involved in the high prevalence of these cases amongst the Kikuyu, Meru and Kamba tribes. The proximity of the hospital to Central and Eastern provinces and the population distribution (Kikuyu, Meru and Kamba tribes forming a large population group in Kenya) may be significant factors which may have contributed to the large number of patients from this group seen in this study. 
The commonest symptoms seen were weakness of the extremities $(96 \%)$ with associated numbness $(36 \%)$, headache $(32 \%)$, neck pain, speech and gait disturbances (Table 1).

Table 1

\begin{tabular}{lcc}
\multicolumn{3}{c}{ Common symptoms in patients with CVA $(n=25)$} \\
\hline Symptom & Frequency & $\%$ \\
\hline Weakness of extremities & 24 & 96 \\
Numbness & 9 & 36 \\
Headache & 8 & 32 \\
Neck pain & 5 & 20 \\
Speech disturbances & 4 & 16 \\
Gait disturbances & 4 & 16 \\
Visual disturbances & 3 & 12 \\
Urinary incontinence & 2 & 8 \\
Impotence & 2 & 8 \\
\hline
\end{tabular}

The duration of these symptoms at the time of diagnosis was between one year and seventeen years. Many of the patients had features suggestive of upper motor neurone lesions i.e. brisk deep tendon reflexes, positive Barbinski and clonus. Other common neurological findings were cerebellar ataxia, muscle paralysis accompanied by wasting and sensory loss, lower cranial nerve palsies and short neck (low hairline) (Table 2).

CT scanning was the most common radiological investigation carried out on patients with suspected CVA at Kenyatta National Hospital (Table 3). Basilar impression was the commonest type of CVA followed by occipitalization of atlas, atlanto-axial dislocation, ArnoldChiari malformation and syringomyelia all in equal frequencies (Table 4). The commonest combined lesions

Table 2

Common neurological findings in patients with CVA $(n=25)$

\begin{tabular}{lcc}
\hline Neurological finding & Frequency & $\%$ \\
\hline Power loss/paralysis & 15 & 60 \\
Sensory loss & 10 & 40 \\
Brisk deep tendon reflexes & 19 & 76 \\
Positive Barbinsk & 9 & 36 \\
Clonus & 7 & 28 \\
Increased muscle tone & 4 & 16 \\
Muscle wasting & 12 & 48 \\
\hline
\end{tabular}

Table 3

Common radiological investigations done in patients with CVA at $\mathrm{KNH}$

\begin{tabular}{lrr}
\hline Investigation & Number of patients & $\%$ \\
\hline Plain X-rays & 14 & 56 \\
Tomography & 3 & 12 \\
Myelography & 9 & 36 \\
CT scan & 20 & 80 \\
MRI & 0 & 0
\end{tabular}

Figure 1

Age and sex distribution in patients $(n=25)$ with craniovertebral anomalies at KNH, 1988-1994.

Table 4

Various types of CVA seen in patients at the $K N H$

\begin{tabular}{lcc}
\hline Type of CVA & No. of patients & $\%$ \\
\hline Basilar impression & 12 & 48 \\
Occipitalization of Atlas & 7 & 28 \\
Atlanto axial dislocation & 7 & 28 \\
Arnold-chiari Malformation & 5 & 20 \\
Syringomyelia & 5 & 20 \\
\hline
\end{tabular}

which were observed in eleven of the patients who underwent surgery, were basilar impression and occipitalization of the atlas. Thirty patients underwent surgery. Two patients died following surgery. Forty percent of the patients improved following surgery, while forty eight percent did not show any improvement. Most of the patients who improved had been symptomatic for less than three years. The common surgical procedures done were posterior fossa decompression, upper cervical laminectomy and placement of vertriculoperitoneal shunt.

\section{DISCUSSION}

Craniovertebral anomaly (CVA) is a rare condition world-wide with most series reporting a few number of cases seen over a long period(2-4). A previous study at the Kenyatta National Hospital reported 27 patients with CVA seen between 1981 and 1987, a period of seven years(5). In this study 38 patients with CVA were seen in the same hospital between 1988 and 1994. Sixty five patients with CVA were therefore seen in the hospital between 1981 and 1994. The increase in the number of cases seen in this study as compared with the earlier study in the same hospital can be attributed to the introduction of the CT scanner which may have contributed to earlier detection of symptomatic patients with subsequent definitive treatment. The results of this study confirm CVA to be an important clinicopathological condition at the Kenyatta National 
Hospital where an average of six cases were seen per year. CVA was slightly more common in male patients according to the findings in this study (male to female ratio of $2: 1$ ) although an earlier study in the same institution had reported a male to female ratio of $2: 3$. The combined results of the two studies show an equal male to female ratio in cases of CVA seen at the Kenyatta National Hospital between 1981 and 1994, findings which compare with those observed elsewhere(7). The age distribution observed in this study was from 10 years to 50 years with a peak in the third decade(4). Similar findings have been reported by other workers. DeBarrors in his study of patients with CVA seen in Brazil reported an age distribution that ranged from 10 to 60 years with a peak in the third decade(4). In Europe the age distribution observed was between 10 years and 80 years with a peak in the fifth decade. The slight variation seen between the results of age distribution in this study and those reported from the Western World may be due to the lower life expectancy found in Kenya. Most of the patients seen at the Kenyatta National Hospital were from Kikuyu, Embu, Meru and Kamba tribes, which are found in the Central part of Kenya. These are Bantu tribes and are traditionally agriculturalists.

The rest of the patients were from the Maasai and Kisii tribes. No cases were reported from the Coastal tribes of Taita, Taveta, Mijikenda, Pokomo and Swahili. Also no cases were seen from Somali, Luhya and Luo tribes of Kenya. CVA is therefore seen mainly in the high altitude highland areas of Kenya according to this study, and no cases were seen from the Coastal region, Lake Victoria region and the arid and semi-arid areas of the country. The average duration from the time of onset of symptoms to the time of presentation in hospital was three years. Patientssought medical treatment at a rather late stage and this may account for the fact that only forty percent of the patients improved following surgery and this did not arrest the progression of the disease in twelve percent of the patients. The commonest mode of presentation was progressive weakness of the extremities (96\%), sensory loss (36\%), headache (32\%), gait disturbances (16\%), neck pain (20\%), and speech disturbances (16\%).

DeBarros in the study of CVA patients done in Brazil reported that the most commonly observed symptoms and signs were progressive limb weakness (68\%), sensory loss $(43 \%)$, dizziness $(37 \%)$ and neck pain $(28 \%)(4)$. Forty percent of the patients with CVA seen in this study were observed to have short neck with restriction in neck movements being reported in $8 \%$ of them. Seventy eight percent of the patients with CVA seen in the Brazil study had short neck, 53\% had restricted neck movements and $68 \%$ had features of craniofacial asymmetry(4).

The radiological investigations done to confirm the diagnosis of CVA in this study were plain x-rays of the skull and cervical spine $(56 \%)$, tomography studies of the craniovertebral junction (12\%), myelography (36\%) and CT scan and CT myelography of cervical spine and head (80\%). An earlier study done at the Kenyatta National Hospital reported that radiological investigations done to confirm CVA included plain x-rays of head and neck $(100 \%)$, tomography of craniovertebral junction (52\%), myelography $(41 \%)$, angiography $(11 \%)$ and ventriculography (7\%). The CT scan has therefore become the commonest radiological investigation in patients with CVA seen at the Kenyatta National Hospital. It has the capacity of revealing Arnold Chiari malformations, associated hydrocephalus and in cases of atlanto-axial dislocation secondary to rheumatoid arthritis it can reveal retro transverse ligament granulomas impinging on the $\operatorname{cord}(6)$.

The most common type of lesions in this study were basilar impression (48\%) followed by atlanto-axial dislocation (28\%) and occipitalization of the atlas (28\%). A previous study in the same hospital reported occipitalization of the atlas as the commonest condition $(74 \%)$ followed by basilar impression $(41 \%)$ and atlanto axial dislocation $(33 \%)(5)$. In other studies elsewhere basilar impression either in isolation or in association with other malformations was observed in $80 \%$ of the patients and occipitalization of the atlas was seen in $30 \%$ of the patients(7). Forty percent of the patients who underwent surgery improved while further progression of the disease process was arrested in another forty eight percent.

Similar findings were observed in the previous study at Kenyatta National Hospital(5). In that study one third of the patients improved following surgery and further clinical deterioriation was prevented in another one third. CVA is therefore an important clinical entity in Kenya found mainly amongst certain ethnic groups in high altitude areas of the country. Most of the patients present late for treatment and therefore less than fifty percent improve following surgery. Greater emphasis should therefore be directed towards early diagnosis of this condition so as to obtain better results after surgery.

\section{ACKNOWLEDGEMENTS}

We gratefully acknowledge the cooperation and assistance of the Director, Kenyatta National Hospital and the Research and Ethics Committee, Kenyatta National Hospital for permission to carry out this study and publish the findings.

\section{REFERENCES}

1. Betrand, G. Anomalies of craniovertebral junction in "Neurological Surgery". Ed. Julian Youmans. Pub W.B Saunders 1982; 1482-1506.

2. Chopra, J.S. Sawhney, I.M., Kak, V.K. and Khosla, V.K. Craniovertebral anomalies: a study of 82 cases. Brit. J. Neurosurg. 1988; 2:455-64.

3. Sood, S., Mahapatra, A.K. and Bhatia, R. Craniovertebral anomaly; effect of surgical management. J. Neurol. Neurosurg. Psychiat. 1992 55:609-12.

4. Debarros, M.C., Ferias W. Ataide, L. and Lins, S. Basilar impression and Arnold Chiari malformation. A study of 66 Cases. J. Neurol, Neurosurg and Psychiat. 1968; 31:596-605.

5. Maranya, G.O. Craniovertebral anomalies at Kenyatta National Hospital. M.Med. Thesis 1988. University of Nairobi.

6. Kaiser, M.C. Veiga-pires, J.A. and Capesius, U.S.P. Atlanto-axial impaction and compression of medulla oblongata and proximal spinal cord in rheumatoid arthritis evaluated by CT scanning. Brit. J. Radiol. 1983; 56:764-766.

7. Schmidt, H. and Sator, K., Bone malformations of the Craniocervical region in "Handbook of clinical Neurology". E.d P.J. Vinken and G.N. Bruyn. Pub.- E. Servier/North Holland. Biomedical press 1978 page 180 . 